[2] Arshad A, Kong KO. Awareness and perceptions of fibromyalgia syndrome: a survey of Malaysian and Singaporean rheumatologists. Singapore Med J. 2007;48:25-30.

[3] Arshad A, Kong KO, Ooi KK. Awareness and perceptions of fibromyalgia syndrome: a survey of southeast asian rheumatologists. J Clin Rheumatol. 2007;13:59-62.

[4] Bloom S, Ablin JN, Lebel D, Rath E, Faran Y, Daphna-Tekoah S, et al. Awareness of diagnostic and clinical features of fibromyalgia among orthopedic surgeons. Rheumatol Int. 2013;33:927-31.

[5] Clark P, Paiva ES, Ginovker A, Salomón PA. A patient and physician survey of fibromyalgia across Latin America and Europe. BMC Musculoskelet Disord. 2013;14:188.

Disclosure of Interests: : None declared

DOI: 10.1136/annrheumdis-2020-eular.5213

\section{THU0468 THERAPIES IN RHEUMATIC AND MUSCULOSKELETAL DISEASES: AN OVERVIEW OF SYSTEMATIC REVIEWS INFORMING THE 2020 EULAR RECOMMENDATIONS FOR INTRA-ARTICULAR THERAPIES INCLUDING SYNOVIORTHESIS}

S. C. Rodriguez-García ${ }^{1}$, R. Castellanos-Moreira ${ }^{2}$, J. Uson Jaeger ${ }^{3}$, E. Naredo ${ }^{4}$, L. Carmona ${ }^{5}$ on behalf of the EULAR Intra-articular Therapies Task Force. ${ }^{1}$ Hospital de La Princesa, Madrid, Spain; ${ }^{2}$ Hospital Clínic de Barcelona, Barcelona, Spain; ${ }^{3}$ Hospital de Móstoles, Móstoles, Spain; ${ }^{4}$ Hospital Universitario Fundación Jiménez Díaz, Madrid, Spain; ${ }^{5}$ InMusc Instituto de Salud Musculoesquelética, Madrid, Spain

Background: Intra-articular therapy (IAT) is subject to wide variability and there are gaps in the evidence on its efficacy and safety.

Objectives: To assess the efficacy and safety of frequently used IATs to inform a EULAR Taskforce.

Methods: We performed an overview of systematic reviews (SR) of randomised clinical trials (RCT) assessing efficacy and safety of IAT in adults with RMDs. MEDLINE was searched until January 2019. SRs were assessed with the AMSTAR-2 tool. Critically low-confidence SRs were excluded.

Results: Of 159 articles identified, 42 were reviewed in detail and 15 met the inclusion criteria (146 RCTs). The populations included were mainly knee osteoarthritis (OA) in $10 \mathrm{SRs}$, rheumatoid arthritis (RA) in 3, hip and temporo-mandibular (TM) OA and shoulder adhesive capsulitis in $1 \mathrm{SR}$ each.

In knee OA, Hyaluronic Acid (HA) showed a modest benefit over placebo for pain and function but with unclear clinical significance in some studies. Platelet rich plasma (PRP) showed a small effect over HA only for function. Mesenchymal stem cells (MSC) performed better than PRP and HA for some outcomes, however, in studies with high risk of bias. Intra-articular Glucocorticoids (GC) were better than placebo (PBO) for pain and function.

More adverse events $(A E)$ were seen in the PRP group compared with $\mathrm{HA}$ and for $\mathrm{HA}$ compared with $\mathrm{PBO}$ including serious $\mathrm{AE}$ each in $1 \mathrm{SR}$ on knee OA. Results for other included diseases are shown in table 1.

Conclusion: Most of the SRs assessed had results of low confidence. HA and GC showed a small, short term benefit in knee arthritis in OA and RA compared to PBO. High risk of bias prevents conclusions on the efficacy of PRP and MSC in knee OA. More AE were reported in PRP and HA treated groups.

Disclosure of Interests: : Sebastian C Rodriguez-García Speakers bureau: Novartis Farmaceutica, S.A., Merck Sharp \& Dohme España, S.A., Sanofi Aventis, UCB Pharma, Raul Castellanos-Moreira Speakers bureau: Lilly, MSD, Sanofi, UCB, Jacqueline Uson Jaeger: None declared, Esperanza Naredo: None declared, Loreto Carmona Grant/research support from: Novartis Farmaceutica, SA, Pfizer, S.L.U., Merck Sharp \& Dohme España, S.A., Roche Farma, S.A, Sanofi Aventis, AbbVie Spain, S.L.U., and Laboratorios Gebro Pharma, SA (All trhough institution)

DOI: 10.1136/annrheumdis-2020-eular.645

\section{THU0469 \\ QUANTIFYING THE PLACEBO EFFECT AFTER INTRA- ARTICULAR INJECTIONS: IMPLICATIONS FOR TRIALS AND PRACTICE}

S. C. Rodriguez-García ${ }^{1}$, R. Castellanos-Moreira ${ }^{2}$, J. Uson Jaeger $^{3}$, E. Naredo ${ }^{4}$, L. Carmona ${ }^{5}{ }^{1}$ Hospital de La Princesa, Madrid, Spain; ${ }^{2}$ Hospital Clínic de Barcelona, Barcelona, Spain; ${ }^{3}$ Hospital Universitario de Móstoles, Móstoles, Spain; ${ }^{4}$ Hospital Universitario Fundación Jiménez Díaz, Madrid, Spain; ${ }^{5}$ InMusc Instituto de Salud Musculoesquelética, Madrid, Spain

Background: In recent years, diverse compounds for intra-articular administration were brought into the market with a subsequent significant and heterogeneous literature production. Understanding the efficacy of intra-articular therapies
(IAT) on pain implies bearing in mind the related placebo (PBO) effect. To date, most studies analyzing it were focused on the compound being administered rather than the route of administration.

Objectives: We aimed at evaluating the size of the PBO effect after intra-articular injections.

Methods: We conducted an overview of systematic reviews (SRs) including randomized-controlled trials (RCTs) of frequently used IAT. SRs with a saline solution PBO arm and high-confidence results according to the AMSTAR-2 tool were selected for analysis.

Data on the change in pain in the PBO arms from baseline to 3-6 and 12-16 weeks after the IA procedure was extracted. The standardized mean differences (SMD) from baseline were calculated as the ratio between the size of the intervention effect in each study and the variability observed in that study. A meta-analysis was then performed using an inverse-variance random-effects model in Review Manager 5.3. The overall effect sizes obtained refer to versions of the SMD, which corresponds to the Hedges' (adjusted) g. e.g. a "g" of 1 indicates the two groups being compared differ by 1 standard deviation and so on. Results: Two SR were included comprising 50 RCTs, 44 not meeting inclusion criteria were excluded so pain, measured by visual analogue scale (VAS) and Lequesne index, was retrieved from 6 RCT.

At 3-6 weeks, an SMD $[95 \% \mathrm{Cl}]=0.74[0.47-1.00]$ was found. One study showing too large an effect was excluded after conducting sensitivity analysis resulting in a significant reduction of heterogeneity with an SMD $=0.62$ [0.45-0.79] (Fig.1). At $12-16$ weeks, we found a SMD $=0.33$ [0.13-0.52] (Fig.2)

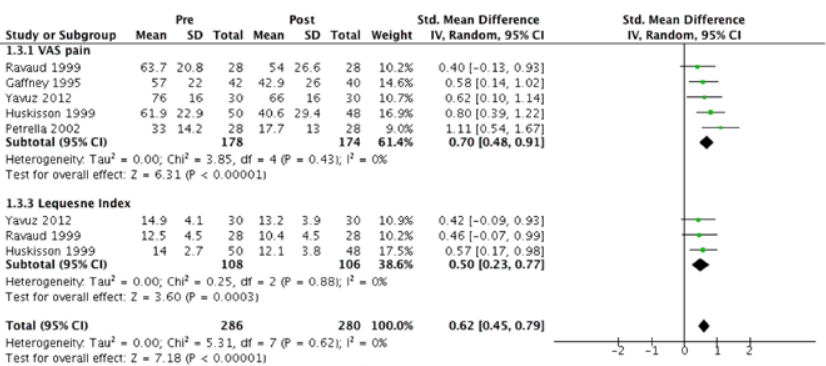

Figure 1. Forest plot for intra-articular PBO effect at 3-6 weeks after injection.

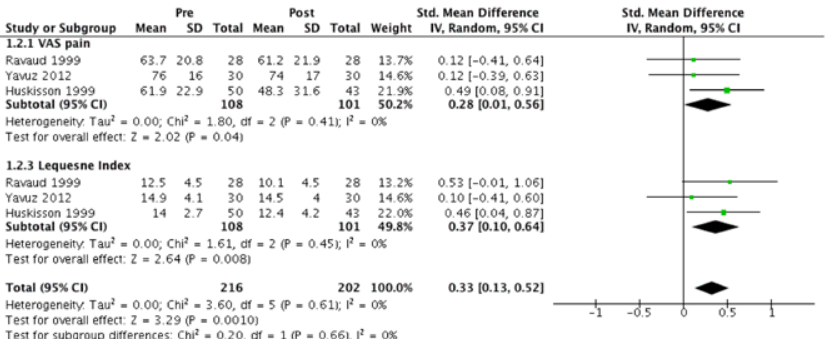

Figure 2. Forest plot for intra-articular PBO effect at 12-16 weeks after injection.

Using the interpretation suggested by Cohen ${ }^{1}$, our results would confirm a moderate to large effect of IA saline (PBO) at 3-6 weeks with a subsequent reduction to a small but persistent effect at 12-16 weeks.

Conclusion: Our results showed a moderate to large short-term effect of intra-articular PBO that persisted on the mid-term although reduced. Based on these findings we suggest this effect should be considered when assessing the efficacy of IAT in RCTs and also in clinical practice where it could be maximized as well.

\section{References:}

[1] Cohen J. Statistical Power Analysis in the Behavioural Sciences (2nd edition). Hillsdale (NJ): Lawrence Erlbaum Associates, Inc., 1988.

Disclosure of Interests: : Sebastian C Rodriguez-García Speakers bureau: Novartis Farmaceutica, S.A., Merck Sharp \& Dohme España, S.A., Sanof Aventis, UCB Pharma, Raul Castellanos-Moreira Speakers bureau: Lilly, MSD, Sanofi, UCB, Jacqueline Uson Jaeger: None declared, Esperanza Naredo: None declared, Loreto Carmona Grant/research support from: Novartis Farmaceutica SA, Pfizer, S.L.U., Merck Sharp \& Dohme España, S.A., Roche Farma, S.A Sanofi Aventis, AbbVie Spain, S.L.U., and Laboratorios Gebro Pharma, SA (All trhough institution)

DOI: 10.1136/annrheumdis-2020-eular.644 